\title{
Diabetes mellitus referida: incidência e determinantes, em coorte de idosos do município de São Paulo, Brasil, Estudo SABE - Saúde, Bem-Estar e Envelhecimento
}

\author{
Reported diabetes mellitus: incidence and determinants in cohort \\ of community dwelling elderly people in São Paulo City, Brazil: \\ SABE study, health, wellness and aging
}

\author{
Manuela de Almeida Roediger ${ }^{1}$ \\ Maria de Fátima Nunes Marucci ${ }^{1}$ \\ Luis Alberto Gobbo ${ }^{2}$ \\ Daiana Aparecida Quintiliano Scarpelli Dourado ${ }^{1}$ \\ Jair Licio Ferreira Santos ${ }^{3}$ \\ Yeda Aparecida de Oliveira Duarte ${ }^{4}$ \\ Maria Lúcia Lebrão ${ }^{5}$
}

\footnotetext{
${ }^{1}$ Departamento de Nutrição, Faculdade de Saúde Pública (FSP), Universidade de São Paulo (USP). Av. Dr. Arnaldo 715, Cerqueira César. 01246904 São Paulo SP Brasil. manuela@usp.br ${ }^{2}$ Departamento de Educação Física, Universidade Estadual Paulista. Presidente Prudente SP Brasil. ${ }^{3}$ Faculdade de Medicina de Ribeirão Preto, USP. Ribeirão Preto SP Brasil. ${ }^{4}$ Departamento de Enfermagem MédicoCirúrgica, Escola de Enfermagem, USP. São Paulo SP Brasil.

${ }^{5}$ Departamento de Epidemiologia, FSP, USP. São Paulo SP Brasil.
}

\begin{abstract}
To verify the association between the incidence of DM and predictors, in a cohort of elderly people. Elderly people ( $\geq 60 y)$ were analyzed, of both genders, participants of the $S A B E$ Survey, carried out in the city of São Paulo, Brazil, in $2000(n=2,143)$ and $2006(n=1,115)$. The study variables were: DM; demographic (gender, age group, education level, companionship in the residence), nutritional status (risk for obesity, body obesity, and high abdominal fat), clinical (number of reported diseases), and lifestyle (alcohol consumption, smoking, intake of meat and fruit and vegetables). Multiple logistic regression $(p<0.05)$ was used to verify the association between variables of this study, with the statistical software Stata/SE 10.1. In 2006, 914 subjects, survivors of 2000, were analyzed and 72 were identified as new cases of DM (7.7/1.000 person-years). It was found that body obesity $(\mathrm{OR}=1.67, \mathrm{CI}=$ 1.00 to 2.81) and high abdominal fat $(O R=2.32$, $C I=1.47$ to 3.67$)$ were predictors of the incidence of DM in the elderly $(p<0.000)$. It was concluded that body obesity and abdominal fat are the variables which contribute to the development of DM in the elderly.
\end{abstract}

Key words Incidence, Diabetes mellitus, Elderly, Predictors
Resumo Verificar a associação da incidência de diabetes melito (DM) e variáveis determinantes em coorte de idosos. Foram analisados idosos, participantes do Estudo SABE - Saúde, Bem-Estar e Envelhecimento, realizado no município de São Paulo, em 2000 (2.143 idosos), e em 2006 (1.115 idosos). As variáveis de estudo foram: DM; sociodemográficas (sexo, grupo etário, escolaridade, companhia no domicílio), estado nutricional (risco para obesidade, obesidade e gordura abdominal), clinicas (número de doenças referidas) e de estilo de vida (ingestão de bebida alcoólica, hábito de fumar, ingestão de carnes e de frutas, legumes e verduras). Utilizou-se regressão logística múltipla para verificar a associação entre variáveis de estudo, utilizando o programa Stata/SE 10.0. Em 2006, 914 idosos, sobreviventes de 2000, foram analisados, dos quais 72 constituiram os novos casos de DM (incidência de 7,7/1.000 pessoas-ano). Verificou-se associação $(p<0,000)$ entre incidência de DM com obesidade corporal ( $O R=1,67 ;$ IC $=1,00-2,81)$ e gordura abdominal elevada $(O R=$ 2,32; IC = 1,47-3,67). Concluiu-se que a obesidade corporal e a gordura abdominal elevada foram as variáveis que contribuem para o desenvolvimento da DM em idosos.

Palavras-chave Incidencia, Diabetes melito, Idosos, Preditores 


\section{Introdução}

O envelhecimento populacional tem sido acompanhado pelo aumento da incidência e da prevalência de doenças e agravos não transmissíveis (DANT), que proporcionam maior ônus para os indivíduos, famílias e Estado, e constituem a principal causa de morbidade, incapacidades e mortalidade da população idosa, em todas as regiões do mundo, tornando-se desafio para países em desenvolvimento, como o Brasil ${ }^{1,2}$. Dentre as doenças crônicas mais incidentes na população idosa brasileira, destaca-se a diabetes melito (DM) tipo $2^{3}$.

Estudos mostram que a prevalência e a incidência de DM têm apresentado tendência de crescimento. Biggs et al. ${ }^{4}$, estudaram uma coorte de idosos ( $\geq 65$ anos) norte-americanos com seguimento de 12,4 anos e constataram incidência de DM de 7,1 /1000 pessoas-anos. Segundo esses autores a incidência de DM dobrou nos últimos 15 anos e é maior nos indivíduos de 65 a 79 anos de idade. Wild et al. ${ }^{5}$ verificou que nos países da América Latina e Caribe, o número de idosos ( $\geq 65$ anos) com DM aumentará em 2030 para $194 \%$. No Brasil, de acordo com os dados da Vigilância de fatores de risco e proteção para doenças crônicas por inquérito telefônico (VIGITEL ${ }^{6}$, representativo das capitais brasileiras e do Distrito Federal, 21\% dos idosos $\geq 65$ anos referiram ter DM.

A literatura científica confirma evidências em relação aos principais fatores que predispõem para a ocorrência da DM na população adulta, entretanto, ainda há controvérsias sobre quais os fatores determinantes estão associados ao desenvolvimento da DM, em idosos. Estudos mostram que o avançar da idade per se tem sido considerado condição independente para diminuição da tolerância à glicose e, consequentemente, para o desenvolvimento da DM em idosos ${ }^{7,8}$. Entretanto, outros fatores, tais como nível de escolaridade, inatividade física, presença de outras morbidades, alimentação inadequada, obesidade, gordura intra-abdominal, tabagismo e o uso abusivo de bebidas alcoólicas, também estão associados ao risco de $\mathrm{DM}^{8-10}$.

O conhecimento dos principais determinantes da DM é imprescindível para compor políticas e projetos voltados para diminuir a ocorrência desse problema de saúde pública na população, principalmente em indivíduos vivendo na comunidade, cenário de atuação propício para propor ações preventivas para controlar esses determinantes que podem contribuir negativa- mente no estado de saúde ${ }^{11}$. Segundo Silva et al. ${ }^{12}$ a presença de doença crônica neste segmento da população é influenciada por fatores de risco que podem ser evitados ou controlados, desde que sejam reconhecidos pelo indivíduo e pelos profissionais de saúde.

Estudos epidemiológicos sobre DM no Brasil têm sido cada vez mais explorados, principalmente, com a população jovem e adultos. Entretanto, estudos para conhecer a incidência de DM, com seguimento da mesma população, considerando indivíduos idosos, vivendo em domicílio, ainda é incipiente. Dessa forma, este estudo tem como objetivo verificar quais os fatores determinantes que contribuem para o desenvolvimento da DM, em coorte de idosos brasileiros domiciliados.

\section{Métodos}

\section{Delineamento e população de estudo}

Trata-se de estudo longitudinal e de associação, com utilização de dados do Estudo SABE: Saúde, Bem-estar e Envelhecimento, realizado no município de São Paulo-SP/Brasil, em 2000 e em 2006.

Em 2000, o Estudo SABE foi coordenado pela Organização Pan Americana de Saúde (OPAS) e caracterizou-se como inquérito multicêntrico, epidemiológico, internacional e de base domiciliar, em sete países da América Latina e Caribe (Argentina, Barbados, Brasil, Chile, Cuba, México e Uruguai), com objetivo de avaliar as condições de vida e estado de saúde de idosos da América Latina ${ }^{13}$.

No Brasil, foi realizado no município de São Paulo, em 2000 e em 2006, com objetivo de verificar as alterações nas condições de vida e de saúde dos idosos e seus fatores determinantes, em função do tempo. Foi coordenado por docentes da Faculdade de Saúde Pública (FSP) da Universidade de São Paulo (USP) e financiado pela Fundação de Amparo à Pesquisa do Estado de São Paulo (FAPESP) e Ministério da Saúde ${ }^{14}$.

A população do estudo SABE, em 2000 foi constituída por 2.143 idosos ( $\geq 60$ anos), de ambos os sexos, residentes no município de São Paulo, que concordaram em participar. O processo de amostragem desse estudo foi realizado de forma probabilística, por meio do método de amostragem por conglomerados em dois estágios. Foi considerada a amostra do primeiro estágio, os 72 setores censitários cadastrados no Departamento de Epidemiologia da FSP da USP, 
sorteados sob o critério de probabilidade proporcional ao número de domicílios, a partir dos 263 setores censitários da Pesquisa Nacional de Amostra de Domicílios (1995), de áreas urbanas do município de São Paulo, correspondendo a 1.568 indivíduos entrevistados ${ }^{13,14}$.

O segundo estágio foi composto por 575 indivíduos, que residiam próximo aos setores selecionados ou, no máximo, dentro dos limites dos distritos aos quais pertenciam os setores sorteados, correspondente ao acréscimo efetuado para compensar a menor densidade populacional de idosos com 75 anos e mais, e a maior taxa de mortalidade dos homens, totalizando 2.143 entrevistas domiciliares ${ }^{13,14}$.

Em 2006, foram novamente reavaliados os indivíduos entrevistados em 2000, correspondendo a 1.115 idosos ( $\geq 65$ anos), que concordaram em participar, novamente do estudo. A redução do número de idosos no período do estudo (2000 a 2006) ocorreu devido a óbitos (649), recusas (178), não localizados (139), mudança de domicílio (51), e institucionalizações $(11)^{14}$.

O questionário utilizado para a coleta de dados do estudo SABE foi proposto pela OPAS, traduzido e adaptado para utilização no Brasil. A obtenção dos dados foi realizada por entrevistadores treinados em duas etapas: 1) visita domiciliar, realizada por um entrevistador, abrangendo questões sobre o estado de saúde dos idosos; 2) visita domiciliar, realizada por dois entrevistadores, responsáveis pelas mensurações antropométricas, e os testes de equilíbrio, flexibilidade e força. Os dados coletados foram revistos por grupo técnico especializado da FSP/USP ${ }^{13}$.

O Estudo SABE foi aprovado pela Comissão Nacional de Ética em Pesquisa - CONEP e pelo Comitê de Ética em Pesquisa - COEP da FSP da USP. Os idosos, que concordaram em participar, assinaram Termo de Consentimento Livre e Esclarecido, em 2000, e em 2006.

Para este trabalho, dos 2.143 idosos entrevistados em 2000, 1.747 referiram não ter recebido diagnóstico de DM, no Estudo SABE. Dos que referiram não ter recebido o diagnóstico de DM, em 2000, foram reavaliados 914 , em 2006, tendo em vista a perda de pessoas, devido a óbitos (649) e de idosos (184) que não tiveram a medida de circunferência da cintura, de peso e altura realizadas.

\section{Variáveis de estudo}

A variável dependente foi diabetes melito, no período de 6 anos de estudo (2000-2006), identificada, pela questão "Alguma vez um médico ou enfermeiro lhe disse que o (a) Sr (a) tem diabetes melito, quer dizer, níveis altos de açúcar no sangue?". As alternativas de resposta eram: sim, não, não sabe, não respondeu, sendo que as duas últimas foram consideradas missing.

Para verificar os determinantes da incidência de DM na população de estudo foram selecionadas variáveis sociodemográficas, estado nutricional, clínicas e estilo de vida, do estudo SABE em 2000, que correspondem a questões utilizadas na literatura científica internacional, para avaliação das hipóteses causais para desenvolvimento dessa doença. Optou-se pela análise dessas variáveis, previamente à referência de ter a doença, com o objetivo de verificar o efeito destas no período de 6 anos.

As variáveis sociodemográficas foram sexo, grupos etários, escolaridade e companhia no domicílio, que foram referidas pelos idosos, identificadas e categorizadas em variáveis dicotômicas, respectivamente, como: Qual o sexo do entrevistado? (Homem e mulher); Quantos anos completos o(a) $\operatorname{Sr}$ (a) tem? (60 a 74 e $\geq 75$ anos); Qual a última série, na escola, o(a) $\mathrm{Sr}$ (a) obteve com aprovação? (< 8 e $\geq 8$ anos de estudo) e Atualmente o(a) $\mathrm{Sr}$ (a) vive sozinho(a) ou acompanhado(a)? (sozinho e acompanhado).

Quanto as variáveis do estado nutricional e clínicas utilizaram-se o risco para obesidade, a obesidade corporal, a gordura abdominal, e o número de doenças referidas (hipertensão arterial sistêmica, doenças cardíacas e acidente vascular cerebral), respectivamente.

O risco para obesidade $\left(\geq 28 \mathrm{e}<30 \mathrm{~kg} / \mathrm{m}^{2}\right)$ e a obesidade corporal (IMC $\geq 30 \mathrm{~kg} / \mathrm{m}^{2}$ ) foram identificadas pelo índice de massa corporal (IMC = peso $-\mathrm{kg}$ / estatura ${ }^{2}-\mathrm{m}$ ) e classificados, segundo a OPAS ${ }^{15}$, sendo consideradas essas classificações como possíveis causas para desenvolvimento da DM. Já a gordura abdominal foi identificada pelos valores de circunferência da cintura (elevado quando, $\mathrm{CC} \geq 102 \mathrm{~cm}$, para homens, e $\geq 88 \mathrm{~cm}$, para mulheres) propostos pela $\mathrm{WHO}^{16}$. O número de doenças foi referido pelo idoso e classificado em 0-2 e 3 ou mais doenças.

$\mathrm{O}$ peso foi medido em balança portátil (SECA, Alemanha), com capacidade de $150 \mathrm{~kg}$ e sensibilidade de $1 \mathrm{~kg}$. Para a medida da circunferência da cintura utilizou-se fita métrica inelástica. As técnicas para mensuração foram padronizadas, segundo Frisancho ${ }^{17} \mathrm{e}$ as medidas foram realizadas em triplicata, utilizando a média de valores para as análises.

Com relação as variáveis de estilo de vida utilizaram-se, também, informações referidas e 
categorizadas em variáveis dicotômicas como: ingestão de bebida alcoólica nos últimos três meses ( $\operatorname{sim} /$ não), hábito de fumar atualmente ( $\operatorname{sim} /$ não), ingestão pelo menos três porções de carnes (bovina, suína, aves e peixes) por semana ( $\operatorname{sim} /$ não); e ingestão de pelo menos duas porções de frutas, legumes e verduras (FLV) ao dia ( $\operatorname{sim} /$ não).

\section{Procedimentos estatísticos}

A população deste estudo é proveniente de processo de amostragem complexa, e, portanto foram utilizados testes estatísticos indicados para estudos do tipo survey. A frequência relativa de todas as variáveis corresponde à frequência ponderada pelo setor censitário ao qual o idoso pertencia.

Para calcular a incidência, foi considerada a DM referida, em 2006. O tempo de observação (6 anos) foi determinado para os sobreviventes, considerando o tempo entre a $1^{\text {a }}$ entrevista de $2000(\mathrm{n}=2143)$ e a última de $2006(\mathrm{n}=1115)$ e de maneira específica para cada caso, uma vez que houve óbitos e perdas no período das entrevistas. Os óbitos ocorridos até a conclusão da coleta de dados do Estudo SABE 2006 foi de 649, sendo 560 casos com data conhecida e 89 com data atribuída para aqueles casos em que a data do óbito era desconhecida. As perdas ocorridas durante o período foram de 379, incluindo 178 indivíduos que recusaram a entrevista (considerou-se o tempo decorrido entre a $1^{\text {a }}$ entrevista de 2000 e a data da 2a entrevista de 2006, em que houve a recusa), e 139 indivíduos não localizados, 51 que mudaram para outro município e 11 institucionalizados (considerou-se a média de dias entre a data da $1^{\text {a }}$ entrevista, em 2000, e a segunda entrevista em 2006.

A associação entre a variável dependente e as independentes foi verificada pelo teste de Rao \& Scott e regressão logística múltipla, com nível de significância de 5\%. A medida da magnitude de efeito foi verificada pelos valores de odds ratio (OR), e respectivos intervalos de confiança (IC 95\%).

Pela análise univariada, foram selecionadas as variáveis com $\mathrm{p}<0,20$, em ordem crescente de entrada, para compor o modelo final de regressão múltipla, permanecendo as variáveis com $\mathrm{p}$ $<0,05$, ou aquelas que alteraram em, no mínimo $10 \%$, o valor de odds ratio. A colinearidade foi verificada pelos valores da Variance Inflation Factor (VIF) - fator de inflação da variância, considerando ausência de colinearidade, quando VIF estivesse entre 0,19 e 5,30. O sexo e os grupos etários foram mantidos para ajuste do modelo, independente do valor de $\mathrm{p}$.

Os cálculos foram realizados pelo programa estatístico Stata Versão 10.1 (Stata Corp., College Station, Estados Unidos).

\section{Resultados}

Dos 914 indivíduos reavaliados em 2006, 842 referiram não ter DM (60\% mulheres) e 72 referiram ter (58\% mulheres), constituindo os novos casos da doença (Tabela 1). A taxa de incidência de DM em 6 anos de estudo foi de 7,7/1000 pessoas-ano.

Em relação as variáveis sociodemográficas verificou-se que aqueles que desenvolveram DM, no período estudado, $58,1 \%$ foram mulheres, $85,7 \%$ constituem o grupo de $60-74$ anos, $80,5 \%$ relataram $<8$ anos de estudo e mais de $80 \%$ viviam acompanhados (Tabela 1 ).

Quanto ao estado nutricional, observouse que dentre os novos casos da doença, 59,2\% apresentaram risco para obesidade, $30,8 \%$ foram classificados como obesos, $67,9 \%$ com excesso de gordura abdominal.

De acordo com as variáveis clínicas e de estilo de vida, verificou-se que $71,4 \%$ relataram ter mais 3 ou mais doenças crônicas, $40 \%$ referiram ingerir de bebidas alcoólicas, 18,5\% são fumantes, $8,9 \%$ e $12,9 \%$ referiam não ingerir carnes e frutas, legumes e verduras (FLV), respectivamente (Tabela 1).

De todas as variáveis analisadas verificou-se que o risco para obesidade, a obesidade corporal, a gordura abdominal elevada e três ou mais doenças referidas foram aquelas que apresentaram associação $(\mathrm{p}<0,20)$ com desenvolvimento da DM em idosos (Tabela 2).

Pela análise da regressão logística, as variáveis que se mantiveram no modelo final e apresentaram associação direta e positiva para incidência de DM foram a obesidade corporal e a gordura abdominal elevada, constituindo as variáveis determinantes para a ocorrência dessa doença em idosos, independente do sexo e grupos etários. Idosos obesos e com excesso de gordura abdominal apresentaram 1,67 e 2,33 mais chances, respectivamente, de desenvolver DM em 6 anos de estudo ( $p<0,05)$. Não foi observada multicolinearidade entre as variáveis estudadas (Tabela 3 ). 
Tabela 1. Distribuição dos idosos segundo ano de estudo, diabetes melito e variáveis sociodemográficas, do estado nutricional, clínicas e de estilo de vida. Estudo SABE, São Paulo-SP/Brasil.

\begin{tabular}{|c|c|c|c|}
\hline \multirow{3}{*}{ Variáveis Analisadas } & \multirow{2}{*}{$\begin{array}{c}2000 \\
\text { Geral (N 1467) }\end{array}$} & \multicolumn{2}{|c|}{2006} \\
\hline & & $\operatorname{Sim}(\mathbf{N} 72)$ & Não (N 842) \\
\hline & $\%$ & $\%$ & $\%$ \\
\hline \multicolumn{4}{|l|}{ Sóciodemográficas } \\
\hline \multicolumn{4}{|l|}{ Sexo } \\
\hline Homem & 41,0 & 41,9 & 39,6 \\
\hline Mulher & 58,0 & 58,1 & 60,4 \\
\hline \multicolumn{4}{|l|}{ Grupos etários } \\
\hline $60-74$ & 78,5 & 85,7 & 83,2 \\
\hline 75 e mais & 21,5 & 14,3 & 16,8 \\
\hline \multicolumn{4}{|l|}{ Escolaridade } \\
\hline$<8$ anos & 83,3 & 80,5 & 77,6 \\
\hline$\geq 8$ anos & 16,7 & 19,5 & 22,4 \\
\hline \multicolumn{4}{|l|}{ Companhia no domicílio } \\
\hline Sozinho & 13,2 & 15,3 & 12,6 \\
\hline Acompanhado & 86,8 & 84,7 & 87,4 \\
\hline \multicolumn{4}{|l|}{ Estado Nutricional } \\
\hline \multicolumn{4}{|l|}{ Risco para obesidade corporal } \\
\hline $\operatorname{Sim}$ & 68,2 & 59,2 & 67,7 \\
\hline Não & 31,8 & 40,8 & 32,3 \\
\hline \multicolumn{4}{|l|}{ Obesidade corporal } \\
\hline Não & 79,5 & 69,2 & 79,0 \\
\hline Sim & 20,5 & 30,8 & 21,0 \\
\hline \multicolumn{4}{|l|}{ Gordura abdominal elevada } \\
\hline Não & 54,9 & 32,1 & 48,1 \\
\hline $\operatorname{Sim}$ & 45,1 & 67,9 & 51,9 \\
\hline \multicolumn{4}{|l|}{ Clínicas } \\
\hline \multicolumn{4}{|l|}{ Número de doenças referidas } \\
\hline $0-2$ & 39,4 & 28,6 & 43,2 \\
\hline 3 e mais & 60,6 & 71,4 & 56,8 \\
\hline \multicolumn{4}{|l|}{ Estilo de vida } \\
\hline \multicolumn{4}{|l|}{ Ingestão de bebida alcoólica } \\
\hline Não & 78,6 & 60,0 & 65,8 \\
\hline Sim & 21,4 & 40,0 & 34,2 \\
\hline \multicolumn{4}{|l|}{ Hábito de fumar } \\
\hline Não & 83,3 & 81,5 & 85,0 \\
\hline $\operatorname{Sim}$ & 16,7 & 18,5 & 15,0 \\
\hline \multicolumn{4}{|l|}{ Ingestão de carnes } \\
\hline Sim & 91,9 & 91,1 & 92,5 \\
\hline Não & 8,1 & 8,9 & 7,5 \\
\hline \multicolumn{4}{|l|}{ Ingestão de FLV } \\
\hline Sim & 82,5 & 87,1 & 82,1 \\
\hline Não & 17,5 & 12,9 & 17,9 \\
\hline
\end{tabular}

Nota: SABE - Saúde, Bem Estar e Envelhecimento; N - número; FLV - frutas, legumes e verduras.

\section{Discussão}

O presente estudo foi desenvolvido visando preencher uma lacuna na área científica, pois, apesar de existirem programas, políticas e ações voltadas para controle e tratamento do diabetes melito na população brasileira, os resultados apresentados destacam elevada taxa de incidência de DM na população idosa brasileira, em relação a países, também, em desenvolvimento ${ }^{18,19}$, contudo é semelhante ou inferior a outros estudos realizados em países desenvolvidos ${ }^{20,21}$, conforme a classificação de desenvolvimento de países proposta pela World Economic Outlook Report ${ }^{22}$.

Neste estudo a taxa de incidência de DM, no período de 6 anos de estudo, foi de 7,7/1000 
Tabela 2. Análise univariada da associação entre incidência de diabetes melito e variáveis estudadas. Estudo SABE, São Paulo-SP/Brasil.

\begin{tabular}{|c|c|c|c|}
\hline Variáveis analisadas & OR & IC (95\%) & p \\
\hline \multicolumn{4}{|l|}{ Sexo } \\
\hline Homem & 1 & \multirow{2}{*}{$0,52-1,58$} & \multirow{2}{*}{0,739} \\
\hline Mulher & 0,91 & & \\
\hline \multicolumn{4}{|l|}{ Grupo etário } \\
\hline 60-74 anos & 1 & \multirow{2}{*}{$0,43-1,54$} & \multirow{2}{*}{0,522} \\
\hline$\geq 75$ anos & 0,81 & & \\
\hline \multicolumn{4}{|l|}{ Escolaridade } \\
\hline$>8$ anos & 1 & \multirow[t]{2}{*}{$0,95-1,08$} & \multirow[t]{2}{*}{0,582} \\
\hline$\leq 8$ anos & 1,01 & & \\
\hline \multicolumn{4}{|l|}{ Companhia no domicílio } \\
\hline Acompanhado & 1 & \multirow[t]{2}{*}{$0,55-2,82$} & \multirow[t]{2}{*}{0,582} \\
\hline Sozinho & 1,25 & & \\
\hline \multicolumn{4}{|l|}{ Risco para obesidade } \\
\hline Não & 1 & \multirow{2}{*}{$0,89-2,32$} & \multirow{2}{*}{0,130} \\
\hline Sim & 1,44 & & \\
\hline \multicolumn{4}{|l|}{ Obesidade corporal } \\
\hline Não & 1 & \multirow{2}{*}{$1,00-2,81$} & \multirow{2}{*}{0,050} \\
\hline Sim & 1,67 & & \\
\hline \multicolumn{4}{|l|}{ Gordura abdominal elevada } \\
\hline Não & 1 & \multirow[t]{2}{*}{$1,23-3,11$} & \multirow[t]{2}{*}{0,005} \\
\hline $\operatorname{Sim}$ & 1,96 & & \\
\hline \multicolumn{4}{|c|}{ Número de doenças referidas } \\
\hline $0-2$ & 1 & \multirow{2}{*}{$0,96-3,73$} & \multirow[t]{2}{*}{0,063} \\
\hline 3 e mais & 1,89 & & \\
\hline \multicolumn{4}{|c|}{ Ingestão de bebida alcoólicas } \\
\hline Não & 1 & \multirow{2}{*}{$0,68-2,15$} & \multirow{2}{*}{0,328} \\
\hline Sim & 1,18 & & \\
\hline \multicolumn{4}{|l|}{ Hábito de fumar } \\
\hline Não & 1 & \multirow[t]{2}{*}{$0,70-2,32$} & 0,403 \\
\hline $\operatorname{Sim}$ & 1,29 & & \\
\hline Ingestão de carnes & & & \\
\hline Não & 1 & $0,43-3,30$ & 0,712 \\
\hline Sim & 1,20 & & \\
\hline Ingestão de FLV & & & \\
\hline Não & 1 & $0,27-1,63$ & 0,376 \\
\hline Sim & 0,67 & & \\
\hline
\end{tabular}

Nota: SABE - Saúde, Bem Estar e Envelhecimento; OR - odds ratio; IC - intervalo de confiança; FLV - frutas, legumes e verduras; negrito $\mathrm{p}<0,20$. Teste de Rao \& Scott $t^{22}$.

pessoas-ano, correspondentes a 72 casos novos. Pesquisa realizada na Jamaica, com 728 indivíduos adultos e idosos identificou 51 novos casos de DM, em 4 anos de estudo, correspondendo a $1,84 / 1000$ pessoas-ano ${ }^{18}$. Outro estudo desenvolvido na Costa Rica com 7039 indivíduos adultos e idosos verificaram uma incidência de DM de $1,62 / 1000$ pessoas-ano ${ }^{19}$.

Rockwood et al. ${ }^{20}$ analisaram uma coorte de 9008 idosos ( $\geq 65$ anos), do Canadian Study of
Tabela 3. Associação entre diabetes melito, em 6 anos de estudo, e variáveis do estado nutricional. Estudo SABE, São Paulo-SP/Brasil.

\begin{tabular}{lcccc}
\hline $\begin{array}{c}\text { Variáveis } \\
\text { analisadas }\end{array}$ & OR & IC (95\%) & $\begin{array}{c}\text { p da } \\
\text { variável }\end{array}$ & $\begin{array}{c}\text { p do } \\
\text { modelo }\end{array}$ \\
\hline $\begin{array}{l}\text { Obesidade } \\
\text { corporal }\end{array}$ & & & & \\
$\quad$ Não & 1 & $1,00-2,81$ & 0,005 & \\
$\quad$ Sim & 1,67 & & & 0,000 \\
Gordura & & & & \\
abdominal & & & & \\
elevada & & & & \\
$\quad$ Não & 1 & $1,47-3,67$ & 0,001 & \\
$\quad$ Sim & 2,33 & & & \\
\hline
\end{tabular}

Nota: SABE - Saúde, Bem Estar e Envelhecimento; OR - odds ratio; IC - intervalo de confiança; negrito $\mathrm{p}<0,05$. Regressão logística múltipla ajustada por sexo e grupos etários.

Health and Aging (CSHA-1), e constaram que a taxa de incidência de DM, 8,6/1000 pessoas-ano, em 4,6 anos de estudo, similar ao observado neste estudo. Entratanto, outro estudo realizado na Alemanha com 6.012 indivíduos adultos e idosos verificaram quantidade elevada de novos casos (401) de DM, em 9 anos de estudo ${ }^{21}$.Ressalta-se que todas essas pesquisas citadas consideraram a informação referida dessa doença, conforme foi utilizado neste estudo.

Estudo de metanálise investigou os preditores da incidência de DM em 32 estudos realizados com indivíduos adultos e idosos em vários países e constatou diferentes taxas de incidência dessa doença entre as regiões analisadas. Nessa pesquisa os novos casos de DM foram analisados segundo a média geométrica por regiões; sendo possível constatar que a taxa de incidência de DM (7,7/1000 pessoas-ano) deste estudo é semelhante à de países europeus (7,2/1000 pessoas-ano), superior aos países asiáticos $(5,2 / 1000$ pessoas -ano) e inferior aos Estados Unidos (13,5/1000 pessoas-ano ${ }^{23}$.

Esses resultados são preocupantes, especialmente nos países em desenvolvimento, como o Brasil e especificamente em idosos, os quais estão mais suscetíveis aos agravos de saúde, e, portanto, com menos condições para enfrentá-los. A presença da DM de acordo com alguns estudos pode diminuir a qualidade de vida e a capacidade funcional para o desempenho de atividades básicas e instrumentais de vida diária, e aumentar os riscos para complicações clínicas, para internações hospitalares e até mesmo óbito nesse grupo etário ${ }^{24}$.

Apesar de não ter sido constatada diferença estatística entre sexo e grupos etários neste es- 
tudo, a taxa de incidência de DM foi maior em mulheres, e no grupo etário 60-74, para ambos os sexos, assim como em outros estudos ${ }^{18,25}$. Outros estudos constataram relação inversa, verificando maior incidência de DM em homens ${ }^{26,27}$. Uma das razões que podem explicar este resultado é que as mulheres buscam assistência médica, mais frequentemente, do que os homens, possibilitando diagnóstico precoce, além de alterações hormonais durante período da menopausa, propiciando o uso de estrógenos e, consequentemente, aumento do peso corporal e da gordura abdominal, fatores que predispõem ao desenvolvimento dessa doença ${ }^{27}$.

Outra possível explicação para a menor incidência da doença no grupo $\geq 75$ anos, pode estar relacionada ao viés de sobrevivência, uma vez que, os idosos mais vulneráveis às complicações da doença, teriam maior probabilidade de morrer, prematuramente ${ }^{28}$. Lebrão e Duarte ${ }^{14}$ constataram que a DM foi a única doença crônica cuja frequência diminuiu com o avançar da idade, em idosos paulistanos. Segundo esses autores, os óbitos decorrentes das complicações da doença podem ter contribuído para esse resultado.

No presente estudo, não houve diferença estatística entre variáveis sociodemográficas e referência de $\mathrm{DM}$, indicando que, nesta população, a incidência da doença não é influenciada por essas variáveis conforme evidenciado por outros autores $^{29}$. Entretanto, Barceló et al. ${ }^{30}$ verificaram que a ocorrência de diabetes melito (15,7\%) esteve associada, positivamente, com baixos níveis de educação ( 0 a 6 anos de escolaridade).

Verificou-se que o risco para obesidade, não apresentou associação com DM, semelhante a outras pesquisas realizadas ${ }^{29,30}$. Esses resultados indicam que estar acima do peso adequado para estatura, não constitui risco para o desenvolvimento de DM em idosos, apesar de ser condição que predispõe à ocorrência da doença, em indivíduos adultos ${ }^{31}$. Diferentemente, neste estudo verificou-se que idosos obesos apresentaram maior probabilidade para o desenvolvimento da DM. Essas evidências confirmam a hipótese de que existem diferenças, quanto ao impacto do estado nutricional e desenvolvimento de doenças crônicas entre indivíduos adultos e idosos, contudo ainda é uma área pouco estudada, com carência de resultados conclusivos e que requer mais pesquisas para investigar essa relação.

Estudo de caráter, também, longitudinal realizado em Barbados-Caribe, com indivíduos obesos, de 40 a 84 anos, utilizando a informação referida de DM, assim como neste estudo, verifica- ram associação positiva e significativa entre DM e obesidade ( $4^{\mathrm{a}}$ quartil, quando IMC $\geq 29,8 \mathrm{~kg} / \mathrm{m}^{2}$ ), sendo que o risco para desenvolver DM, em nove anos de estudo é de 3,7 vezes maior para aqueles que estão obesos ${ }^{32}$. No Brasil, não há estudos dessa natureza que investigam essa relação, porém Passos et al. ${ }^{29}$ avaliaram idosos de Minas Gerais do Projeto Bambuí (Estudo Saúde e Envelhecimento) e verificaram que a prevalência de DM pode estar associada a obesidade $\left(\geq 30 \mathrm{~kg} / \mathrm{m}^{2}\right)$.

Neste estudo foi constatado que a gordura abdominal é uma variável que contribui para o aumento do número de casos de DM em idosos, sendo aquela a que apresentou maior risco para desenvolvimento dessa doença em 6 anos de estudo, da mesma maneira como tem sido observado, também, em indivíduos adultos, sendo um determinante comum entre ambas os grupos populacionais ${ }^{18,23}$. Pesquisa longitudinal realizada com indivíduos de 25 a 74 anos, da Jamaica, utilizando a informação referida da DM, como neste estudo, verificaram que idosos com excesso de gordura abdominal apresentaram maior risco para o desenvolvimento da $\mathrm{DM}^{18}$.

Existem poucos estudos com idosos, que realizaram essa análise. Srikanthan et al. ${ }^{33}$, que avaliou idosos de uma coorte participantes do The MacArthur Successful Aging Study, verificaram que o desenvolvimento da DM ocorre especificamente em indivíduos com excesso de gordura na região abdominal, independente do sexo e grupo etário, conforme constatado nesta pesquisa. Até o momento não há estudos longitudinais com idosos brasileiros apresentando essas informações, o que realça a importância deste estudo como sendo, provavelmente o primeiro estudo brasileiro a investigar os fatores que determinam o desenvolvimento da DM nesse grupo etário.

Uma das limitações metodológicas deste estudo é o fato de que as estimativas de incidência de DM são baseadas em dados referidos feitos em relação ao tempo de desenvolvimento da doença. É possível que os dados relativos a incidência de DM possam estar subestimados, por não conseguir autorrelato da condição, mesmo quando ela está presente. Por outro lado, a informação referida da DM, sem testes clínicos para comprovar o diagnóstico da doença, é válida e pode ser utilizada, conforme outros autores já verificaram ${ }^{34}$.

Outra limitação refere-se a perda de seguimento e a ausência de conhecimento de quando os dados foram censurados, situações essas que podem interferir na estimativa da taxa de incidência. Além disso, não é possível estabelecer se houve mudanças ao longo do tempo, quanto as 
variáveis explicativas, tendo em vista que os dados dos indivíduos participantes foram obtidos apenas em dois momentos (2000 e 2006). Ressalta-se, ainda, as diferenças de idade, escolaridade, de etnia, de delineamentos das pesquisas e de valores de referência de IMC e de CC utilizados, que podem interferir na comparação entre os estudos e dificultar na identificação do real efeito desses indicadores sobre a incidência de DM.

No entanto, considerando a incidência significativa de DM e seus efeitos negativos, e dada a escassez de informações sobre esta condição e determinantes associados em idosos no Brasil, este estudo é um contributo relevante para o alargamento da compreensão da DM nesse grupo etário e formulação de futuros estudos brasileiros no sentido de diminuir a ocorrência dessa doença na população.

Medidas preventivas, como monitoramento da glicemia, controle do peso corporal, alimentação adequada, e práticas regulares de atividade física constituem as principais recomendações utilizadas em pesquisas, clínicas e centros de saú- de para evitar o desenvolvimento da DM. Entretanto, a identificação e o acompanhamento de variáveis corporais, como a presença de obesidade corporal e, especialmente a de gordura abdominal devem ser implementadas em protocolos de atendimento ao idoso, em diferentes áreas de atenção, tendo em vista que esse grupo populacional futuramente constituíra o cenário epidemiológico brasileiro.

\section{Conclusão}

Baseada nas considerações apresentadas foi possível constatar que a obesidade corporal e a gordura abdominal foram as variáveis que contribuiram para o desenvolvimento da DM em idosos. Essas informações são válidas e merecem atenção pelos pesquisadores e profissionais da área, no sentido incitar discussões para avaliar se as estratégias implementadas contemplam esses determinantes analisados e se essas têm sido eficazes e eficientes para evitar o desenvolvimento dessa doença.

\section{Colaboradores}

MA Roediger realizou a proposta deste trabalho, a organização e a análise dos dados e a redação do manuscrito. MFN Marucci, LA Gobbo, DAQS Dourado e JLF Santos participaram na análise e interpretação dos resultados, da revisão e da aprovação final do manuscrito. YAO Duarte e ML Lebrão participaram da revisão e da aprovação da versão final do manuscrito.

\section{Agradecimentos}

À Fundação de Amparo à Pesquisa do Estado de São Paulo (FAPESP) pelo apoio financeiro prestado. 


\section{Referências}

1. Campolina AG, Dini OS, Ciconelli RM. Impacto da doença crônica na qualidade de vida de idosos da comunidade em São Paulo (SP, Brasil). Cien Saude Colet 2011; 16(6):2919-2925.

2. Centers for Disease Control and Prevention (CDC). The State of Aging \& Health in America 2013. Atlanta: National Center for Chronic Disease Prevention and Health Promotion, Division of Population Health; 2013

3. Instituto Brasileiro de Geografia e Estatística (IBGE). Estudos e Pesquisas, Informações Demográficas e Socioeconômicas, $n^{\circ}$.27. Síntese de Indicadores Sociais: uma análise das condições de vida da população brasileira. Rio de Janeiro: IBGE; 2010.

4. Biggs ML, Mukamal KJ, Luchsinger JA, Ix JH, Carnethon MR, Newman AB, de Boer IH, Strotmeyer ES, Mozaffarian D, Siscovick DS. Association Between Adiposity in Midlife and Older Age and Risk of Diabetes in Older Adults. JAMA 2010; 303(24):2504-2512.

5. Wild S, Roglic G, Green A, Sicree R, King H. Global prevalence of diabetes: estimates for the year 2000 and projections for 2030. Diabetes Care 2004; 27(5):10471053.

6. Brasil. Ministério da Saúde (MS). Vigitel Brasil 2010: Vigilância de fatores de risco e proteção para doenças crônicas por inquérito telefônico. Brasília: MS; 2011.

7. Qin X, Li J, Zhang Y, Ma W, Fan F, Wang B, Xing H, Tang G, Wang X, Xu X, Xu X, Huo Y. Prevalence and Associated Factors of Diabetes and Impaired Fasting Glucose in Chinese Hypertensive Adults Aged 45 to 75 Years. Plos one 2012; 7(8):1-8.

8. Tanaka T, Gjonça E, Gulliford MC. Income, wealth and risk of diabetes among older adults: cohort study using the English longitudinal study of ageing. Eur J Public Health 2011; 22(3):310-317.

9. Chen R, Song Y, Hu Z, Brunner EJ. Predictors of Diabetes in Older People in Urban China. Plos one 2012; 7(11):1-11.

10. Neeland IJ, Turer AT, Ayers CR, Powell-Wiley TM, Vega GL, Farzaneh-Far R, Grundy SM, Khera A, McGuire DK, Lemos JA. Dysfunctional adiposity and the risk of prediabetes and type 2 diabetes in obese adults. JAMA 2012, 308(11):1150-1159.

11. Dorner B, Friedrich EK, Posthauer ME. Practice paper of the American Dietetic Association: Individualized nutrition approaches for older adults in health care communities. J Am Diet Assoc 2010; 110(10):15541563.

12. Silva RCP, Simões MJS, Leite AA. Fatores de risco para doenças cardiovasculares em idosos com diabetes mellitus tipo 2. Rev Ciênc Farm Básica Apl 2007; 28(1):113-121.

13. Lebrão ML, Laurenti R. Saúde, bem-estar e envelhecimento: o estudo SABE no Município de São Paulo. Rev. Bras Epidemiol 2005; 2(8):127-141.

14. Lebrão ML, Duarte YAO. Desafios de um estudo longitudinal: o Projeto SABE. Saúde Coletiva 2008; 5(24):166-167.

15. Organização Pan-Americana da Saúde (OPAS). XXXVI Reunión Del Comitê Asesor de Investgaciones em Salud - Encuesta Multicêntrica - Salud Belnestar y de Envejecimiento (SABE) em América Latina e ela Caribe - Informe preliminar. Brasília: OPAS; 2001.
16. World Health Organization (WHO). Obesity: preventing and managing the global epidemic. Report of a WHO Consultation. Geneva: WHO; 2000. [WHO Technical Report Series 894].

17. Frisancho RA. Anthropometric Standards fort the Assessment of Growth and Nutritional Status. Ann Arbor: The University of Michigan Press, 1990.

18. Sargeant LA, Bennett FI, Forrester TE, Cooper RS, Wilks RJ. Predicting Incident Diabetes in Jamaica: The Role of Anthropometry. Obesity Res 2002; 10(8):792798.

19. Laclé-Murray A, Valero-Juan LF. Incidencia de diabetes tipo 2 en un área urbano marginal de Costa Rica. AMC 2008; 50(1):29-34.

20. Rockwood K, Awalt E, MacKnigh C, McDowell I. Incidence and outcomes of diabetes mellitus in elderly people: report from the Canadian Study of Health and Aging. CMAJ 2000; 162(6):769-772.

21. Meisinger C, Döring A, Thorand B, Heier M, Löwel H. Body fat distribution and risk of type 2 diabetes in the general population: are there differences between men and women? The MONICA/KORA Augsburg Cohort Study. Am J Clin Nutr 2006; 84(3):483-489.

22. World Economic Outlook (International Monetary Fund). World economic outlook: a survey by the staff of the International Monetary Fund. Washington: International Monetary Fund; 2014.

23. Vazquez G, Duval S, Jacobs DR, Silventoinen K. Comparison of Body Mass Index, Waist Circumference, and Waist/Hip Ratio in Predicting Incident Diabetes: A Meta-Analysis. Epidemiol Rev 2007; 29(1):115-128.

24. Sinclair A, Morley J, Rodriguez-Mañas L, Paolisso G, Bayer T, Zeyfang A, Bourdel-Marchasson I, Vischer U, Woo J, Chapman I, Dunning T, Meneilly G, Rodriguez-Saldana J, Gutierrez Robledo LM, Cukierman-Yaffe T, Gadsby R, Schernthaner G, Lorig K. Diabetes Mellitus in Older People: Position Statement on behalf of the International Association of Gerontology and Geriatrics (IAGG), the European Diabetes Working Party for Older People (EDWPOP), and the International Task Force of Experts in Diabetes. J Am Med Dir Assoc 2012; 13(6):497-502.

25. González-Villalpando C, Dávila-Cervantes CA, Zamora-Macorra M, Trejo-Valdivia B, González-Villalpando ME. Incidence of type 2 diabetes in Mexico: Results of The Mexico City Diabetes Study after 18 years of follow-up. Salud pública Méx 2014; 56(1):11-17.

26. Sánchez Martínez M, Blanco A, Castell MV, Gutiérrez Misis A, González Montalvo JI, Zunzunegui MV, Otero Á. Diabetes in older people: Prevalence, incidence and its association with medium- and long-term mortality from all causes. Aten Primaria 2014; 46(7):376-384.

27. McBean AM, Li S, Gilbertson DT, Collins AJ. Differences in diabetes prevalence, incidence, and mortality among the elderly of four racial/ethnic groups: whites, blacks, hispanics, and asians. Diabetes Care 2004; 27(10):2317-2324.

28. Francisco PMSB, Belon AP, Barros MBA, Carandina L, Alves Maria CGP, Goldbaum M, Chester LGC. Diabetes auto-referido em idosos: prevalência, fatores associados e práticas de controle. Cad Saude Publica 2010; 26(1):175-184. 
29. Passos VMA, Barreto SM, Dniz LM, Lima-Costa MF. Type 2 diabetes: prevalence and associated factors in a Brazilian community - the Bambuí Health and Aging Study. Med J 2005; 123(2):66-71.

30. Barceló A, Gregg EW, Valero MP, Robles SC. Waist circunference, BMI and the prevalence of self-reported diabetes among the elderly of the United States and sxi cities of Latin America and the Caribean. Diab Res Cli Pract 2007; 78(3):418-427.

31. Wald NJ, Bestwick JP, Morris JK. Body weight reduction to avoid the excess risk of type 2 diabetes. $\mathrm{Br} \mathrm{J} \mathrm{Gen}$ Pract 2012; 62(599):411-414.

32. Nemesure B, Wu SY, Hennis A, Leske MC. The Relationship of Body Mass Index and Waist-Hip Ratio on the 9-Year Incidence of Diabetes and Hypertension in a Predominantly African-Origin Population. Annals of Epidemiology 2008; 18(8):657-663.

33. Srikanthan P, Seeman TE, Karlamangla AS. WaistHip-Ratio as a Predictor of All-Cause Mortality in High-Functioning Older Adults. Ann Epidemiol 2009; 19(10):724-731.

34. Lima-Costa MF, Peixoto SV, Firmo JOA, Uchoa E. Validade do diabetes auto-referido e seus determinantes: evidências do projeto Bambuí. Rev Saude Publica 2007; 41(6):947-953.

Artigo apresentado em 30/05/2015

Aprovado em 22/11/2016

Versão final apresentada em 24/11/2016 